\title{
THE ANTIOXIDANT ENZYME PEROXIREDOXIN-2 IS DEPLETED IN LYMPHOCYTES SEVEN DAYS AFTER ULTRA-ENDURANCE EXERCISE
}

James E. Turner ${ }^{1^{*}}$, Stuart J. Bennett ${ }^{2^{*}}$, John P. Campbell ${ }^{3}$, Jos A. Bosch ${ }^{4,5}$, Sarah Aldred ${ }^{6}$ and Helen R. Griffiths ${ }^{2^{* *}}$

${ }^{1}$ Department for Health, University of Bath, Bath, BA2 7AY, UK, ${ }^{2}$ Life and Health Sciences, Aston University, Birmingham, B4 7ET, UK, ${ }^{3}$ School of Immunity and Infection, University of Birmingham, UK, ${ }^{4}$ School of Psychology, University of Amsterdam, Amsterdam, 1012WX, The Netherlands, ${ }^{5}$ Mannheim Institute of Public Health, Social and Preventive Medicine (MIPH), Mannheim Medical Faculty, University of Heidelberg, Germany, ${ }^{6}$ School of Sport and Exercise Sciences, University of Birmingham, B15 2TT, UK

**Corresponding Author: Helen R. Griffiths. Life and Health Sciences, Aston University, Birmingham, B4 7ET. Tel: +44 (0)121 204 3950. Email:

H.R.Griffiths@aston.ac.uk. *These authors contributed equally to this work

Short title: Ultra-running and lymphocyte peroxiredoxin-2

\section{ABSTRACT}

Purpose: Peroxiredoxin-2 (PRDX-2) is an antioxidant and chaperone-like protein critical for cell function. This study examined whether the levels of lymphocyte PRDX-2 are altered over one month following ultra-endurance exercise. Methods: Nine middle-aged men undertook a single-stage, multi-day 233 km (145 mile) ultra-endurance running race. Blood was collected immediately before (PRE), upon completion/retirement (POST), and following the race at DAY 1, DAY 7 and DAY 28. Lymphocyte lysates were examined for PRDX-2 by reducing SDS-PAGE and western blotting. In a sub-group of men who completed the race $(n=4)$ PRDX2 oligomeric state (indicative of redox status) was investigated. Results: Ultraendurance exercise caused significant changes in lymphocyte PRDX-2 $\left(F_{(4,32)}\right.$ 3.409, $p=0.020, \eta^{2}=0.299$ ): seven-days after the race, PRDX-2 levels in lymphocytes had fallen to $30 \%$ of pre-race values $(p=0.013)$ and returned to nearnormal levels at DAY 28. Non-reducing gels demonstrated that dimeric PRDX-2 (intracellular reduced PRDX-2 monomers) was increased in 3 of 4 race completers immediately post-race, indicative of an "antioxidant response". Moreover, monomeric PRDX-2 was also increased immediately post-race in 2 of 4 racecompleting subjects, indicative of oxidative damage, which was not detectable by DAY 7. Conclusions: Lymphocyte PRDX-2 was decreased below normal levels 7 
days after ultra-endurance exercise. Excessive accumulation of reactive oxygen species induced by ultra-endurance exercise may underlie depletion of lymphocyte PRDX-2 by triggering its turnover after oxidation. Low levels of lymphocyte PRDX-2 could influence cell function and might, in part, explain reports of dysregulated immunity following ultra-endurance exercise.

Keywords: Oxidative stress, reactive oxygen species, peroxiredoxins, runningexercise human 


\section{INTRODUCTION}

Ultra-endurance exercise is associated with increased biomarkers of inflammation (e.g., the acute phase protein $C$ reactive protein; CRP) and the production of reactive oxygen species (ROS) ${ }^{[1]}$. Although low levels of these highly reactive molecules are important for intracellular signaling, excess ROS can overwhelm antioxidant defences, causing oxidative damage in a variety of tissues, including lymphocytes ${ }^{[2]}$. The present study examined whether peroxiredoxin-2 (PRDX-2), an antioxidant molecule important for immune function, was depleted in lymphocytes over the month following an ultra-endurance running race.

One of the main functions of lymphocytes, the most predominant mononuclear cells in blood, is to provide immune surveillance by detecting and eliminating cells infected with viruses and bacteria, or cells that have undergone a malignant transformation. Following ultra-endurance exercise, lymphocytes exhibit increased oxidative damage as measured by protein oxidation and DNA damage ${ }^{[3,4]}$. Conceivably, exercise-induced oxidative damage in lymphocytes might alter aspects of immunity that rely on redox homeostasis. For example, lymphocyte activation, proliferation and cytotoxicity are impaired following depletion of the peroxide-quenching antioxidant molecule glutathione ${ }^{[5]}$. Moreover, low intracellular antioxidant levels and redox-sensitivity have been implicated in the reactivation of latent viruses ${ }^{[6]}$ and there is substantial evidence that large volumes of exercise are associated with periods of illness ${ }^{[7]}$. Conversely, very low levels of PRDX-2 are associated with uncontrolled immune activation that might exacerbate inflammatory activity following exercise ${ }^{[8-10]}$.

PRDX-2 is one of six isoforms of the peroxiredoxin family, which represent a group of multifunctional antioxidant enzymes, approximately 20-30 kDa in size ${ }^{[11]}$. All peroxiredoxin isoforms share the same antioxidant mechanism; the use of a catalytic cysteine residue to reduce peroxynitrite, hydrogen peroxide and hydroperoxides ${ }^{[12]}$. A major role for reduced peroxiredoxins is the control of redoxdependent intracellular signaling, and therefore a variety of cell functions by regulating hydrogen peroxide levels ${ }^{[13]}$. Indeed, PRDX-2 is the most abundant antioxidant enzyme in the cytosol of lymphocytes, and its levels appear to be involved in the regulation of lymphocyte function ${ }^{[8,14]}$. Conversely, monocytes, a relatively minor proportion of mononuclear cells in blood, contain little or no PRDX-2 [15].

PRDX-2 can exist in various redox states within a cell. Prior to reduction of peroxides, PRDX-2 is present as a reduced monomeric form, and upon reaction with molecules such as hydrogen peroxide, the catalytic cysteine residue becomes oxidized to form sulphenic acid in the first instance. This oxidized PRDX-2 monomer is only present transiently due to the rapid formation of a disulphide bond between the cysteine residue of a neighbouring PRDX-2 subunit, resulting in a dimer. In some circumstances PRDX-2 becomes over-oxidized to form sulphinic acid, inducing a structural transition that serves to induce cell cycle arrest ${ }^{[16]}$. Oxidation 
of the catalytic cysteine residue to sulphinic and sulphonic acids, promotes disruption of alpha helices and the exposure of hydrophobic domains that drive the formation of stable decameric complexes of PRDX-2 ${ }^{[16]}$. In turn, decamers lack peroxidase activity, allow redox signaling and function as molecular chaperones to support the refolding of mildly-oxidized proteins ${ }^{[12]}$.

We have shown previously that the levels of glutathione in whole blood are depleted for up to one month after ultra-endurance exercise ${ }^{[4]}$. Considering the majority of glutathione in whole blood is derived from erythrocytes, it remains largely unexplored whether ultra-endurance exercise depletes antioxidant defences in lymphocytes. In addition, since antioxidant molecules in lymphocytes are important for cell function and therefore immune surveillance, then depletion of these molecules might significantly affect immune function. The present study examined whether PRDX-2 level and oligomerisation is altered in lymphocytes over one month following ultra-endurance exercise.

\section{METHODS}

\section{Ethical approval}

Informed consent was obtained in writing following ethical approval from the School of Sport and Exercise Sciences research ethics committee of the University of Birmingham (approved study number: 08/60). All procedures conformed to the standards set by the Declaration of Helsinki.

\section{Experimental design}

Nine healthy men (mean \pm standard deviation; age $46.1 \pm 5.3$ years, body mass index $24.9 \pm 2.3 \mathrm{~kg} \cdot \mathrm{m}^{-2}$, maximal oxygen consumption $56.3 \pm 3.3 \mathrm{ml} \cdot \mathrm{kg}^{-1} \cdot \mathrm{min}^{-}$ $\left.{ }^{1}\right)$ took part in this study as previously described ${ }^{[4]}$. Participants undertook a singlestage, multi-day $233 \mathrm{~km}$ (145 mile) running event. The race started at 06:00 on Saturday the $23^{\text {rd }}$ of May 2009 , and participants who completed the race, crossed the finish line between 21:15 on Sunday the $24^{\text {th }}$ of May, and 01:50 on Monday the $25^{\text {th }}$ of May. Diet was not controlled for logistical reasons. Participants did not rest during the race, other than for very short periods (e.g., to urinate or change clothing) and none of the participants slept. The typical running strategy adopted by participants consisted of approximately 50-55 minutes of running, with 5-10 minutes of walking (allowing for recovery and nutritional intake), repeated in cycles until the race was completed. Due to exhaustion, the final hour of exercise was typically walking. Blood samples were collected immediately before the race (PRE) and as close to the time of exercise cessation for each participant as was logistically possible, irrespective of whether they retired early or completed the race (POST; 65 \pm 64 minutes after). Additional blood samples were collected following the race at as close to 24 hours after exercise cessation as was logistically possible for each participant (DAY 1; mean $26 \pm 4$ hours) and seven and twenty-eight days later (DAY 7 and DAY 28 respectively). Participants refrained from exercising for at least 7 days after the race. 


\section{Sample preparation}

Plasma and peripheral blood mononuclear cells (PBMCs) were processed within 60 minutes of collection and frozen at $-80^{\circ} \mathrm{C}$ as previously described ${ }^{[4]}$. PBMCs are hereafter referred to as lymphocytes as the results reported below reflect changes in lymphocyte, and not monocyte, PRDX-2 levels: monocytes represent a small proportion of PBMCs and contain little or no detectable PRDX-2 ${ }^{[15]}$. Cell preparations were thawed and lysed using RIPA buffer (Thermo Scientific, UK) containing a protease inhibitor cocktail (Sigma Aldrich, Dorset UK). Total protein concentration was determined using the bicinchoninic acid method.

\section{Analytical procedures}

Plasma CRP was measured using an immunoturbidimetric assay based upon latex particle agglutination (Tina-quant ${ }^{\circledR}$ CRP latex high sensitivity assay, Indianapolis, USA) and measured on an automated clinical chemistry analyser (Roche Hitachi Cobas).

Total PRDX-2 levels and monomeric, dimeric and decameric complexes of PRDX-2 were measured by reducing and non-reducing SDS-PAGE respectively, followed by western blotting. Proteins $(10 \mu \mathrm{g})$ were loaded $1: 1$ in sample buffer (4\% SDS, $20 \%$ glycerol, $0.004 \%$ bromophenol blue and $0.125 \mathrm{M}$ Tris $\mathrm{HCl}, \mathrm{pH} 6.8$ ), with and without $10 \%$ 2-mercaptoethanol, onto 15 or $18 \%$ polyacrylamide gels. Electrophoresis at $115 \mathrm{~V}$ was performed for approximately 1.5 hours using a MiniPROTEAN $^{\circledR}$ tetra-cell (Bio-Rad, Hemel Hempstead, UK) with electrophoresis buffer (25 mM Tris, pH 8.3, $192 \mathrm{mM}$ glycine, and 0.2\% w/v SDS). Separated proteins were transferred to Hybond- ${ }^{\circledR}$ PVDF membrane (GE Healthcare, Amersham, UK) with transfer buffer (25 mM Tris, $\mathrm{pH} 8.3,192 \mathrm{mM}$ glycine, and 40\% w/v methanol) for 2 hours at $170 \mathrm{~mA}$. Membranes were blocked overnight at $4^{\circ} \mathrm{C}$ with $3 \%$ BSA (Sigma Aldrich, Dorset, UK) in TBS, washed three times for 10 minutes in TTBS $(0.05 \%$ Tween-20) and incubated with rabbit polyclonal anti-PRDX-2 antibody (1: 1000, Ab15572, AbCam, Cambridge, UK) or mouse monoclonal anti-actin antibody (1: 10 000, Sigma Aldrich, Dorset, UK) for 2 hours at room temperature. PVDF membranes were washed three times for 10 minutes in TTBS (0.05\% Tween-20). PRDX-2 blots were incubated with a peroxidase conjugated goat anti-rabbit antibody (1: 5000; A6154, Sigma Aldrich, Dorset, UK), and actin blots were incubated with a peroxidase-conjugated goat anti-mouse antibody (1: 25000 , Sigma Aldrich, Dorset, UK) for 2 hours at room temperature. Membranes were washed three times for 10 minutes in TTBS (0.05\%) and visualized using ECL+ chemiluminescent detection system (GE Healthcare, Amersham, UK). Protein bands were scanned using a molecular imager GS-800 calibrated densitometer (Bio-Rad) and quantified using Quantity One ${ }^{\text {TM }}$ software (Bio-Rad, Hemel Hempstead, UK). Densitometry values for each protein band were recorded and expressed as arbitrary units. 


\section{Data analysis}

Data were confirmed as having normal distribution using the KolmogorovSmirnov test. Changes in PRDX-2 level were assessed using Analysis of Variance (ANOVA). Potential confounding factors (e.g., finishing status) were entered into ANOVAs as individual covariates and examined for significance (Analysis of covariance; ANCOVA). Effect sizes are reported as eta-squared $\left(\eta^{2}\right)$. Conventionally, $\eta^{2}$ values of $0.01,0.06$, and 0.14 are considered small, medium and large effect sizes respectively. Post-hoc paired-samples $t$-tests identified differences relative to baseline. Significance was set at $p<0.05$. Data are presented as means \pm standard error of the mean (SEM) unless otherwise stated. Data were analysed using SPSS statistical package version 18.0 for Windows (SPSS Inc., USA).

\section{RESULTS}

\section{Ultra-endurance race}

Four men completed the race (finishers). Table 1 shows that there were no physiological differences between the finishers $(n=4)$ and those who retired prematurely due to exhaustion (non-finishers; $n=5$ ). As finishing status did not influence the results reported below as shown by analysis of covariance, all men were included in the main analyses (i.e., CRP, lymphocyte and monocyte responses, total PRDX-2 level).

\section{Ultra-endurance exercise induces an inflammatory response}

Compared to pre-race, plasma CRP was increased approximately 100 -fold immediately post-race and remained at this level for 24 hours. Seven days after the race, plasma CRP remained approximately 8-fold higher than pre-race levels, returning to normal values within one month of the race (see Figure 1, main effects of time; $\left.F_{(4,32)} 3.409, p<0.001, \eta^{2}=0.921\right)$.

\section{Lymphocyte and monocyte numbers before and after ultra-endurance exercise}

Table 2 shows lymphocyte and monocyte counts, and the ratio between these cells, in samples collected before and after ultra-endurance exercise. Immediately after the race, there was a trend for a fall in the number of lymphocytes (i.e., lymphocytopenia) and a significant increase in the number of monocytes (i.e., monocytosis). Subsequently, there was a trend for a fall in the lymphocyte/monocyte ratio immediately after exercise. In all other post-race samples (i.e., DAY 1, DAY 7 and DAY 28 post-race) lymphocyte and monocyte counts were comparable to pre-race levels. There were no significant differences in lymphocyte and monocyte counts in samples collected from finishers compared to non-finishers (time $\times$ finishing status interactions all; $F_{(4,28)}<1.49, p>0.05$ ). Moreover, controlling statistically for minor fluctuations in the lymphocyte/monocyte ratio between samples did not influence the results reported below, as shown by analysis of covariance (data not shown). 
Lymphocyte PRDX-2 is depleted below normal levels seven days after ultraendurance exercise

Ultra-endurance exercise caused a significant alteration in the level of PRDX-2 in lymphocytes (see Figure 2, main effects of time; $F_{(4,32)} 3.409, p=0.020$, $\eta^{2}=0.299$ ). Compared to baseline there was a $17 \%$ increase in PRDX-2 level immediately after the race (paired samples $t$-test; $t{ }_{(8)}-1.605, p=0.147$ ). Twentyfour hours after exercise the level of PRDX-2 had fallen to $74 \%$ of pre-race values ( $t$ ${ }_{(8)}=0.854, p=0.418$ ). Seven-days after exercise PRDX-2 level fell to $30 \%$ of prerace values (paired samples $t$-test; $t_{(8)} 3.187, p=0.013$ ). Twenty eight days postrace PRDX-2 returned to a similar level to that measured twenty-four hours after the race, which was not significantly different from baseline (76\% of pre-race values; paired samples $t$-test; $t$ (8) $1.037, p=0.330$ ).

\section{Lymphocyte PRDX-2 is over-oxidized after ultra-endurance exercise}

To investigate whether the changes in PRDX-2 level following ultraendurance exercise could be explained by PRDX-2 over-oxidation, samples from a sub-group of men $(n=4)$ were analysed. All men in this sub-group finished the race and their changes in PRDX-2 represented the group as a whole $(n=9)$. Samples were examined from the time points that showed the most pronounced changes in PRDX-2 (i.e., PRE, POST and DAY 7). Statistical analyses were not conducted on these data.

As illustrated in Figure 3, under normal intracellular conditions, PRDX-2 can exist as a monomer, dimer or decamer, dependent on redox-state. Within cells, the monomeric form typically represents reduced PRDX-2. Oxidized PRDX-2 usually forms dimers. However, when ROS are in excess, the already oxidized PRDX-2 becomes over-oxidized and may exist as a monomer or as a decamer ${ }^{[12]}$. Upon cell lysis, reduced monomeric PRDX-2 undergoes mild oxidation and therefore appears as dimers on non-reducing SDS-PAGE. Over-oxidized PRDX-2 usually resolves as a decamer. With excessive ROS production, PRDX-2 monomers are unable to dimerise due to over-oxidation, and therefore remain as monomers on non-reducing SDS-PAGE ${ }^{[14]}$.

Figure $4 \mathrm{~A}$ shows that two bands at approximately $250 \mathrm{kDa}$ and $50 \mathrm{kDa}$ (indicative of decameric and dimeric PRDX-2 respectively) are present at baseline. For subject 1, 2 and 9, the intensity of the $50 \mathrm{kDa}$ band (indicative of reduced/mildly oxidized PRDX-2) increased immediately post-race. Moreover, a band at approximately $25 \mathrm{kDa}$ (indicative of monomeric over-oxidized PRDX-2) appeared immediately post-race in subjects 2 and 9 . At seven days post-race, the band at $25 \mathrm{kDa}$ was absent in all subjects, and there was a noticeable reduction in the intensity of the $50 \mathrm{kDa}$ band in subjects 1,2 , and 9 . There was no noticeable change in intensity of the $50 \mathrm{kDa}$ and $25 \mathrm{kDa}$ bands post-race and seven days post-race compared to baseline in subject 8 .

Densitometry values of each band visible on non-reducing SDS-PAGE were expressed as a ratio of monomeric and decameric PRDX-2 (indicative of overoxidized) to dimeric PRDX-2 (indicative of reduced/mildly-oxidized) (Figure 4B). 
This ratio increased immediately after and seven days post-race, indicative of oxidative stress. Further, when monomeric and decameric PRDX-2 and dimeric PRDX-2 were expressed as a proportion of all PRDX-2 oligomers, there was a clear increase in monomeric and decameric PRDX-2 (indicative of over-oxidized) and a concomitant decrease in dimeric PRDX-2 (indicative of reduced/mildly oxidized) immediately after and seven days post-race. These data suggest that ultraendurance exercise causes over-oxidation of PRDX-2 (Figure 4A).

\section{DISCUSSION}

This study demonstrates that lymphocyte PRDX-2 is depleted to less than one-third of normal levels ( $70 \%$ reduction) seven days after an ultra-endurance running event in nine middle-aged men. Further analyses in a sub-group of men who completed the race $(n=4)$ indicated that in some individuals, PRDX-2 becomes over-oxidised after ultra-endurance exercise and is found predominantly in the decameric or monomeric forms. Whereas over-oxidized monomeric PRDX-2 appears to be cleared within seven days, the over-oxidised decameric form seems to persist for at least seven days after ultra-endurance exercise. This is the first study to examine PRDX-2 levels and redox-state in lymphocytes following exercise.

There was a large decrease in lymphocyte PRDX-2 levels seven days after the race. One possible explanation for this finding is that in response to excessive ROS production, perhaps mediated by post-exercise respiratory burst ${ }^{[10]}$, PRDX-2 becomes over-oxidized to the sulphinic or sulphonic forms which appear as monomers under non-reducing conditions. Although low concentrations of hydrogen peroxide transiently elevate PRDX-2 ${ }^{[17]}$, high concentrations lower PRDX-2 mRNA and protein expression in lymphocytes and cause PRDX-2 over-oxidation ${ }^{[18,19]}$. Over-oxidized PRDX-2 can also form decamers and exhibit chaperone-like activity to protect cellular proteins from denaturation and promote refolding ${ }^{[20]}$. Eventually, over-oxidized PRDX-2 is degraded via the proteasome, the main intracellular pathway responsible for removal of oxidized proteins ${ }^{[21,22]}$ and PRDX-2 levels return to normal or even below normal levels. Our results are consistent with the turnover of over-oxidized PRDX-2 in cells. In an analysis of non-reducing SDSPAGE in a sub-group of men who completed the race $(n=4)$ and were therefore exposed to an equivalent level of exercise stress, we examined the redox status of PRDX-2 at the time points exhibiting the largest changes in total PRDX-2 (i.e., PRE, POST, DAY 7) using non-reducing SDS-PAGE. Our results suggest that overoxidized monomeric PRDX-2, which increased immediately post-race, was absent seven days later, possibly indicating clearance.

A second explanation and possible function of the lower lymphocyte PRDX-2 levels observed seven days after exercise might be related to homeostatic replacement of damaged and/or "senescent" T lymphocytes following exercise. For example, there are reports showing that a small number of lymphocytes (1-10\%) can be damaged by exercise and become apoptotic ${ }^{[23]}$. It is unlikely that this small number of apoptotic cells could account for the changes in levels of PRDX-2 in total 
lymphocytes directly, but PRDX-2 may be down-regulated in undamaged lymphocytes ${ }^{[8,9]}$. Such PRDX-2 down-regulation, which is implicated in lymphocyte proliferation, might function to replace "senescent" cells, such as terminally differentiated virus-specific T lymphocytes, which are characteristic of an ageing adaptive immune system ${ }^{[24]}$. Indeed, it has been proposed that exercise removes excess numbers of virus-specific lymphocytes, that are normally apoptosisresistant, to limit their accumulation during ageing and persistent infection ${ }^{[24]}$. The present results are consistent with this idea, where the down-regulation of PRDX-2 may be an adaptive mechanism underlying the restorative proliferation of "healthy" cells following exercise.

There is debate as to whether the health benefits of regular physical activity extend to extreme forms of exercise. Many studies have examined oxidative damage following ultra-endurance exercise ${ }^{[1]}$, and while the majority of studies report increased biomarkers of oxidative damage in lymphocytes following exercise ${ }^{[3,4]}$, others report little damage or no long lasting damage ${ }^{[25]}$. It has also been argued that the increased ROS production is beneficial, as it results in a characteristic "antioxidant response", whereby the level of protective antioxidant enzymes is transiently elevated ${ }^{[26]}$. In the present study, there was a trend for increased lymphocyte PRDX-2 levels immediately after exercise. One explanation for this observation might be an acute antioxidant response to exercise-induced hydrogen peroxide accumulation ${ }^{[17]}$, which for example, might be caused by superoxide release from mitochondria ${ }^{[27]}$. In support of this hypothesis, it has been shown experimentally that de novo synthesis of PRDX-2 occurs during, or in the hours following, an oxidative stress stimulus ${ }^{[18]}$. In addition, the increase in hydrogen peroxide level might exceed the capacity for removal due to overoxidation of PRDX-2, as shown for subjects 2 and 9 during the course of exercise (Figure 3A). An alternative explanation for the elevated PRDX-2 levels observed in this study might be due to non-specific, exercise induced $T$ lymphocyte activation, since stimulating the $\mathrm{T}$ cell receptor results in hydrogen peroxide-mediated signaling and a subsequent increase in PRDX-2 levels ${ }^{[8]}$. Indeed, the increased expression of lymphocyte cell surface proteins associated with activation during exercise, including CD25 (the IL-2 receptor), CD69 and HLA-DR, suggests that exercise can directly activate $T$ lymphocytes in peripheral blood ${ }^{[28]}$.

The lower PRDX-2 levels in lymphocytes following ultra-endurance exercise in the present study, might be an attractive mechanism to examine in future studies attempting to understand the reports of dysregulated immunity (i.e., inflammatory activity and upper respiratory tract infections; URTI) following extreme exercise ${ }^{[7,29]}$. While glutathione depletion is associated with viral reactivation and impaired lymphocyte function ${ }^{[6]}$, lower than normal levels of PRDX-2 are associated with the reverse: uncontrolled and inappropriate immune activation ${ }^{[8]}$. PRDX-2 is a critical regulator of cell-mediated immunity and animals lacking the PRDX-2 gene show uncontrolled expansion of $\mathrm{CD}^{+} \mathrm{T}$ lymphocytes in models of chronic infection, which results in lethal immunopathology ${ }^{[8]}$. Therefore, low levels of PRDX-2 in CD8 ${ }^{+} \mathrm{T}$ lymphocytes might exaggerate inflammatory responses in the days following 
exercise, considering these cells are potent producers of IFN- - , TNF- $\alpha$ and IL-6 ${ }^{[30,31]}$. In the present study, CRP, an acute phase reactant released from the liver in response to IL-6, was approximately 100-fold higher than normal for at least 24 hours after ultra-endurance exercise. Values close to $100 \mathrm{mg} / \mathrm{L}$, as reported in the present study, are typically associated with major bacterial infections. Seven days after ultra-endurance exercise, CRP was approximately 8-fold higher than normal levels, which returned to pre-race values within one month of the race. Considering CRP has a half life of 4-6 hours, the present results show that ultra-endurance exercise results a temporary period (i.e., 7 days) of inflammation that is akin to inflammatory disorders. Interestingly, individuals with larger-than-normal inflammatory responses to environmental stressors are more likely to exhibit symptoms of URTI, than individuals with normal inflammatory responses ${ }^{[32]}$.

It should be considered that in the present study PRDX-2 was measured in PBMCs, a heterogenous population of cells consisting mostly of lymphocytes but also some monocytes. While the majority of PRDX-2 would have been derived from lymphocytes as monocytes contain little or no PRDX-2 ${ }^{[15]}$, the results could in principle, be influenced by the proportion of each cell type in the samples. For example, in the present study, samples collected immediately post-race contained fewer lymphocytes and more monocytes compared to pre-race samples. Despite normalising for total protein assayed, compared to pre-race, a larger proportion of the protein was derived from monocytes post-race. Thus, it might be expected for the level of PRDX-2 to fall. In the present study however, the opposite was true: there was a trend for an increase in PRDX-2 immediately post-race, presumably evidence of an "antioxidant response". Moreover, the main finding that lymphocyte PRDX-2 is depleted below normal levels seven days after ultra-endurance exercise, is derived from comparing two blood samples collected at rest (i.e., PRE vs. DAY 7 ), in which the proportion of lymphocytes and monocytes were comparable.

The present study describes for the first time the kinetics of PRDX-2 oligomerisation and expression level in lymphocytes following ultra-endurance exercise. Although PRDX-2 has not been examined previously following this type of exercise, increased concentrations of the antioxidant molecule thioredoxin-1 has been reported in plasma immediately after ultra-endurance exercise ${ }^{[33]}$. In the context of the present results, an increase in thioredoxin-1 might be part of the antioxidant response to exercise, which serves to recycle mildly oxidized PRDX-2 following exercise-induced hydrogen peroxide production. In the context of chronic exercise training, PRDX-2 levels have been shown to elevated in erythrocytes ${ }^{[34]}$ but not in muscle ${ }^{[35]}$ after three months of moderate intensity cycling.

Taken together with existing literature, the present results indicate that exercise causes time-dependent changes in the level, redox-state and likely turnover of PRDX-2, which may be a consequence of excess ROS production in lymphocytes. The immunological consequences of these observations remain to be elucidated. 


\section{ACKNOWLEDGEMENTS}

Funding: University of Birmingham UK.

\section{DECLARATION OF INTEREST STATEMENT}

None of the authors declare a conflict of interest.

\section{References}

[1] Knez WL, Coombes JS, Jenkins DG. Ultra-endurance exercise and oxidative damage: implications for cardiovascular health. Sports Med 2006;36:429-441.

[2] Powers SK, Nelson WB, Hudson MB. Exercise-induced oxidative stress in humans: cause and consequences. Free Radic Biol Med 2011;51:942-950.

[3] Tauler P, Sureda A, Cases N, Aguilo A, Rodriguez-Marroyo JA, Villa G, Tur JA, Pons A. Increased lymphocyte antioxidant defences in response to exhaustive exercise do not prevent oxidative damage. $J$ Nutr Biochem 2006;17:665-671.

[4] Turner JE, Hodges NJ, Bosch JA, Aldred S. Prolonged depletion of antioxidant capacity after ultraendurance exercise. Med Sci Sports Exerc 2011;43:1770-1776.

[5] Droge W, Schulze-Osthoff K, Mihm S, Galter D, Schenk H, Eck HP, Roth S, Gmunder H. Functions of glutathione and glutathione disulfide in immunology and immunopathology. FASEB J 1994;8:1131-1138.

[6] Staal FJ, Roederer M, Herzenberg LA, Herzenberg LA. Intracellular thiols regulate activation of nuclear factor kappa $B$ and transcription of human immunodeficiency virus. Proc Natl Acad Sci U S A 1990;87:9943-9947.

[7] Gleeson M, Walsh NP. The BASES expert statement on exercise, immunity, and infection. J Sports Sci 2012;30:321-324.

[8] Michalek RD, Crump KE, Weant AE, Hiltbold EM, Juneau DG, Moon EY, Yu DY, Poole LB, Grayson JM. Peroxiredoxin II regulates effector and secondary memory CD8+ $T$ cell responses. J Virol 2012;86:13629-13641.

[9] Moon EY, Noh YW, Han YH, Kim SU, Kim JM, Yu DY, Lim JS. T lymphocytes and dendritic cells are activated by the deletion of peroxiredoxin II (Prx II) gene. Immunol Lett 2006;102:184-190. 
[10] Cooper DM, Radom-Aizik S, Schwindt C, Zaldivar F, Jr. Dangerous exercise: lessons learned from dysregulated inflammatory responses to physical activity. J Appl Physiol 2007;103:700-709.

[11] Brinkmann C, Brixius K. Peroxiredoxins and sports: new insights on the antioxidative defense. J Physiol Sci 2013;63:1-5.

[12] Wood ZA, Poole LB, Karplus PA. Peroxiredoxin evolution and the regulation of hydrogen peroxide signaling. Science 2003;300:650653.

[13] Kang SW, Chae HZ, Seo MS, Kim K, Baines IC, Rhee SG. Mammalian peroxiredoxin isoforms can reduce hydrogen peroxide generated in response to growth factors and tumor necrosis factoralpha. J Biol Chem 1998;273:6297-6302.

[14] Szabo-Taylor KE, Eggleton P, Turner CA, Faro ML, Tarr JM, Toth S, Whiteman M, Haigh RC, Littlechild JA, Winyard PG. Lymphocytes from rheumatoid arthritis patients have elevated levels of intracellular peroxiredoxin 2, and a greater frequency of cells with exofacial peroxiredoxin 2, compared with healthy human Iymphocytes. Int J Biochem Cell Biol 2012;44:1223-1231.

[15] Van Brussel I, Schrijvers DM, Martinet W, Pintelon I, Deschacht M, Schnorbusch K, Maes L, Bosmans JM, Vrints CJ, Adriaensen D, Cos $\mathrm{P}$, Bult $\mathrm{H}$. Transcript and protein analysis reveals better survival skills of monocyte-derived dendritic cells compared to monocytes during oxidative stress. PLoS One 2012;7:e43357.

[16] Phalen TJ, Weirather K, Deming PB, Anathy V, Howe AK, van d, V, Jonsson TJ, Poole LB, Heintz NH. Oxidation state governs structural transitions in peroxiredoxin II that correlate with cell cycle arrest and recovery. J Cell Biol 2006;175:779-789.

[17] Bast A, Wolf G, Oberbaumer I, Walther R. Oxidative and nitrosative stress induces peroxiredoxins in pancreatic beta cells. Diabetologia 2002;45:867-876.

[18] Rabilloud T, Heller M, Gasnier F, Luche S, Rey C, Aebersold R, Benahmed M, Louisot $\mathrm{P}$, Lunardi J. Proteomics analysis of cellular response to oxidative stress. Evidence for in vivo overoxidation of peroxiredoxins at their active site. J Biol Chem 2002;277:1939619401.

[19] Dahlan HM, Karsani SA, Rahman MA, Hamid NA, Top AG, Ngah WZ. Proteomic analysis reveals that treatment with tocotrienols reverses the effect of $\mathrm{H}(2) \mathrm{O}(2)$ exposure on peroxiredoxin expression in human lymphocytes from young and old individuals. J Nutr Biochem 2012;23:741-751.

[20] Jang HH, Lee KO, Chi YH, Jung BG, Park SK, Park JH, Lee JR, Lee SS, Moon JC, Yun JW, Choi YO, Kim WY, Kang JS, Cheong GW, Yun DJ, Rhee SG, Cho MJ, Lee SY. Two enzymes in one; two yeast peroxiredoxins display oxidative stress-dependent switching from a 
peroxidase to a molecular chaperone function. Cell 2004;117:625635.

[21] Drake SK, Bourdon E, Wehr NB, Levine RL, Backlund PS, Yergey $A L$, Rouault TA. Numerous proteins in Mammalian cells are prone to iron-dependent oxidation and proteasomal degradation. Dev Neurosci 2002;24:114-124.

[22] Friguet $B$. Oxidized protein degradation and repair in ageing and oxidative stress. FEBS Lett 2006;580:2910-2916.

[23] Mars M, Govender S, Weston A, Naicker V, Chuturgoon A. High intensity exercise: a cause of lymphocyte apoptosis? Biochem Biophys Res Commun 1998;249:366-370.

[24] Simpson RJ. Aging, persistent viral infections, and immunosenescence: can exercise "make space"? Exerc Sport Sci Rev 2011;39:23-33.

[25] Reichhold S, Neubauer O, Ehrlich V, Knasmuller S, Wagner KH. No acute and persistent DNA damage after an Ironman triathlon. Cancer Epidemiol Biomarkers Prev 2008;17:1913-1919.

[26] Radak Z, Chung HY, Goto S. Systemic adaptation to oxidative challenge induced by regular exercise. Free Radic Biol Med 2008;44:153-159.

[27] Sahlin K, Shabalina IG, Mattsson CM, Bakkman L, Fernstrom M, Rozhdestvenskaya Z, Enqvist JK, Nedergaard J, Ekblom B, Tonkonogi M. Ultraendurance exercise increases the production of reactive oxygen species in isolated mitochondria from human skeletal muscle. J Appl Physiol 2010;108:780-787.

[28] Bishop NC, Fitzgerald C, Porter PJ, Scanlon GA, Smith AC. Effect of caffeine ingestion on lymphocyte counts and subset activation in vivo following strenuous cycling. Eur J Appl Physiol 2005;93:606613.

[29] Gleeson M, Pyne DB, Austin JP, Lynn FJ, Clancy RL, McDonald WA, Fricker PA. Epstein-Barr virus reactivation and upper-respiratory illness in elite swimmers. Med Sci Sports Exerc 2002;34:411-417.

[30] Effros RB, Dagarag M, Spaulding C, Man J. The role of CD8+ T-cell replicative senescence in human aging. Immunol Rev 2005;205:147157.

[31] Lorre K, Kasran A, Van VF, de BM, Ceuppens JL. Interleukin-1 and B7/CD28 interaction regulate interleukin-6 production by human $T$ cells. Clin Immunol Immunopathol 1994;70:81-90.

[32] Boyce WT, Chesney M, Alkon A, Tschann JM, Adams S, Chesterman B, Cohen F, Kaiser P, Folkman S, Wara D. Psychobiologic reactivity to stress and childhood respiratory illnesses: results of two prospective studies. Psychosom Med 1995;57:411-422.

[33] Marumoto M, Suzuki S, Hosono A, Arakawa K, Shibata K, Fuku M, Goto C, Tokudome Y, Hoshino H, Imaeda N, Kobayashi M, Yodoi J, 
Tokudome S. Changes in thioredoxin concentrations: an observation in an ultra-marathon race. Environ Health Prev Med 2010;15:129134.

[34] Moghaddam DA, Heber A, Capin D, Kreutz T, Opitz D, Lenzen E, Bloch W, Brixius K, Brinkmann C. Training increases peroxiredoxin 2 contents in the erythrocytes of overweight/obese men suffering from type 2 diabetes. Wien Med Wochenschr 2011;161:511-518.

[35] Brinkmann C, Chung N, Schmidt U, Kreutz T, Lenzen E, Schiffer T, Geisler S, Graf C, Montiel-Garcia G, Renner R, Bloch W, Brixius K. Training alters the skeletal muscle antioxidative capacity in noninsulin-dependent type 2 diabetic men. Scand J Med Sci Sports 2012;22:462-470. 


\section{Table Legends}

\section{Table 1}

Characteristics of finishers and non-finishers (mean \pm standard deviation).

\begin{tabular}{|c|c|c|c|}
\hline & Finishers $(n=4)^{\mathrm{a}}$ & Non-finishers $(n=5)^{b}$ & \\
\hline Age (years) & $43 \pm 3$ & $49 \pm 6$ & $p>.05$ \\
\hline Body Mass Index (Kg.m²) & $23.7 \pm 1.3$ & $25.8 \pm 2.7$ & $p>.05$ \\
\hline Body Fat $(\%)$ & $14.0 \pm 1.3$ & $14.2 \pm 3.3$ & $p>.05$ \\
\hline LTPA $^{\mathrm{C}}\left(\mathrm{MET}\right.$.min.week $\left.{ }^{-1}\right)$ & $2837 \pm 911$ & $4116 \pm 931$ & $p>.05$ \\
\hline $\mathrm{VO}_{2} \max \left(\mathrm{ml} \cdot \mathrm{kg}^{-1} \cdot \mathrm{min}^{-1}\right)$ & $58.6 \pm 3.1$ & $54.5 \pm 2.2$ & $p>.05$ \\
\hline Max Heart Rate (bpm) & $174 \pm 4$ & $176 \pm 5$ & $p>.05$ \\
\hline Running distance (km) & $233.4 \pm 0.0$ & $126.7 \pm 29.7$ & $p<.01$ \\
\hline Running duration (hh:mm) & $41: 40 \pm 2: 13$ & $20: 44 \pm 7: 15$ & $p<.01$ \\
\hline Running speed (km/h) & $5.6 \pm 0.7$ & $6.6 \pm 0.7$ & $p>.05$ \\
\hline
\end{tabular}

${ }^{a}$ independent samples $t$-test between finishers and non-finishers
${ }^{b}$ Finishing status did not confound the changes in lymphocyte peroxiredoxin-2 level as determined by analysis of covariance.

${ }^{\mathrm{C}}$ Leisure Time Physical Activity, as assessed by the international physical activity questionnaire (IPAQ)

\section{Table 2}

Lymphocyte and monocyte numbers

\begin{tabular}{llllll}
\hline Cells $\mathbf{x} \mathbf{1 0}^{\mathbf{9}}$ & PRE & POST & DAY 1 & DAY 7 & DAY 28 \\
\hline Lymphocytes & $2.56 \pm 0.82$ & $2.03 \pm 0.73$ & $2.27 \pm 0.50$ & $2.08 \pm 0.44$ & $2.24 \pm 0.54$ \\
Monocytes & $0.34 \pm 0.16$ & $0.68 \pm 0.36^{*}$ & $0.41 \pm 0.27$ & $0.30 \pm 0.09$ & $0.36 \pm 0.21$ \\
Lymph/Mono & $9.22 \pm 6.00$ & $3.92 \pm 2.74$ & $7.84 \pm 4.92$ & $7.49 \pm 2.84$ & $9.53 \pm 7.29$
\end{tabular}

${ }^{*} p<0.05$ paired samples $t$-test compared to PRE 


\section{Figure Ledgends}

\section{Figure 1}

Plasma CRP increases in response to ultra-endurance running $(n=9) .{ }^{* * *} p<0.001$ compared to PRE.

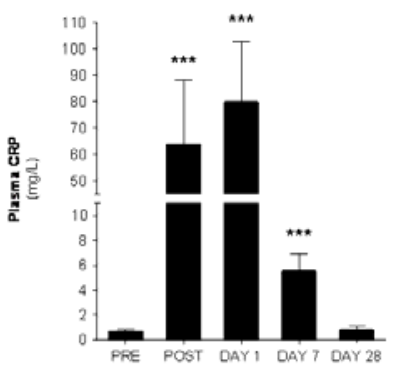

\section{Figure 2}

Total PRDX-2 levels in response to ultra-endurance running. A) Bars represent total PRDX-2 levels in lymphocyte cell lysates $(n=9)$. Error bars indicate mean \pm SEM. PRDX-2 was depleted below pre-race levels seven days after ultraendurance exercise. B) Representative western blots of lymphocyte cell lysates under reducing conditions.
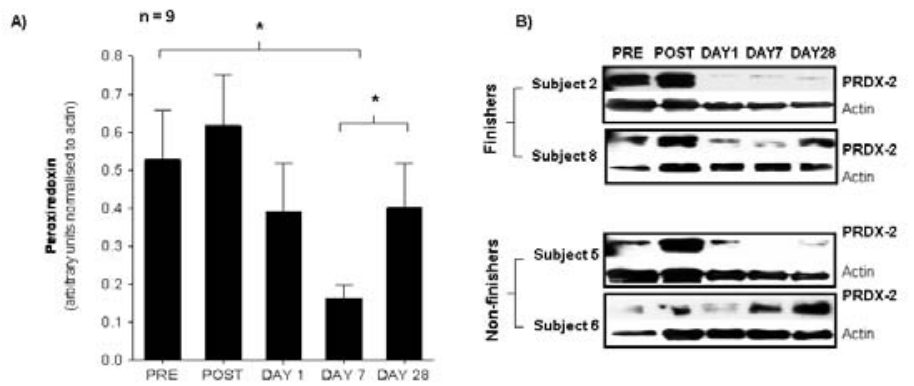


\section{Figure 3}

PRDX-2 oligomeric states. A) Cellular PRDX-2 oligomers: reduced PRDX-2 monomers can be oxidized to sulphenic acid. This mildly oxidized monomer can be attacked by a neighbouring cysteine on a PRDX-2 subunit to form an interdisulphide bond resulting in an oxidized dimer, or over-oxidized to sulphinic or sulphonic acid, which in turn can form over-oxidized decamers. B) PRDX-2 oligomers under non-reducing conditions: upon cell lysis, reduced monomeric PRDX-2 undergoes mild oxidation to sulphenic acid and resolves as dimers (50kDa), whereas over-oxidized PRDX-2 can either exist as a decamer (250kDa) and also as a monomer (25kDa).

A) Cellular PRDX-2 oligomers

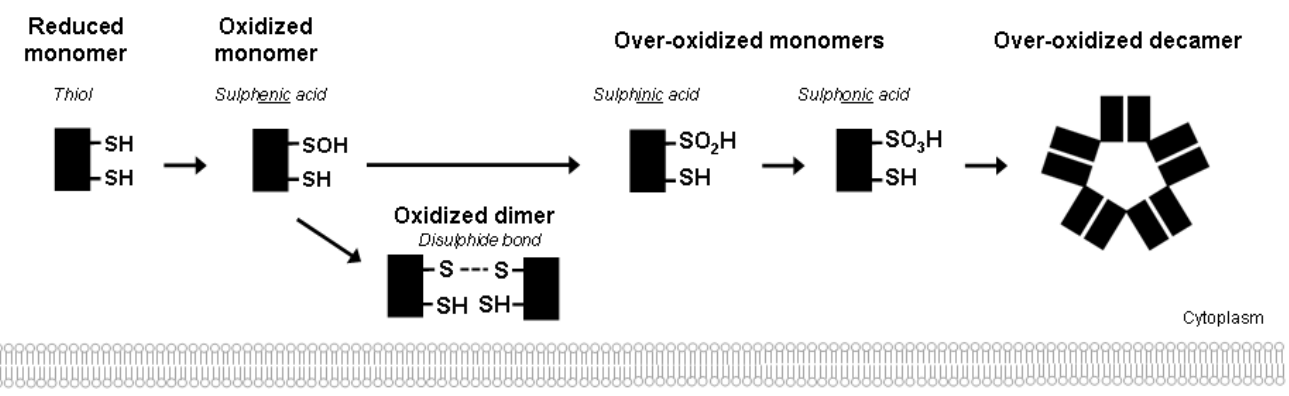

B) PRDX-2 oligomers on non-reducing SDS-PAGE

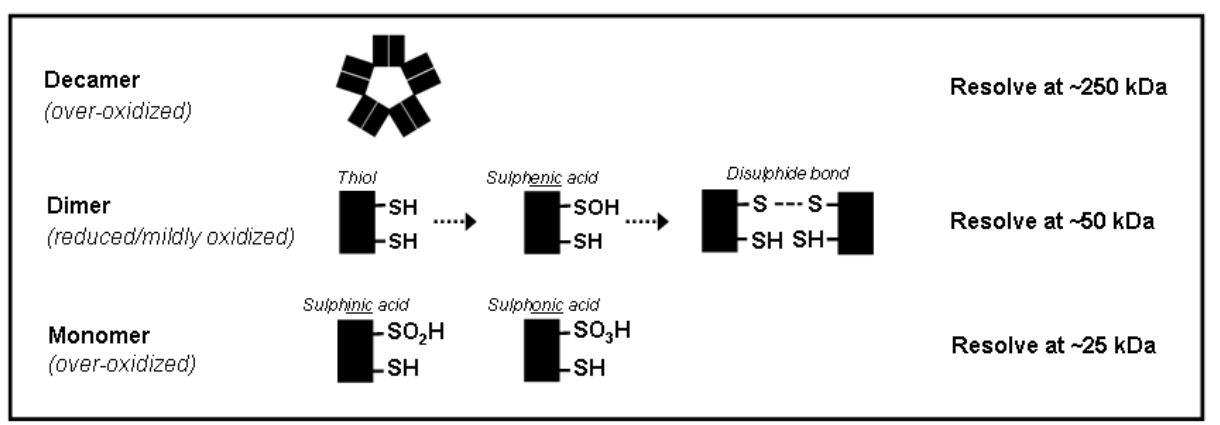

"All forms of PRDX-2 resolve at 25kDa on a reducing SDS-PAGE 


\section{Figure 4}

PRDX-2 redox state in response to ultra-endurance running $(n=4$; finishers only). A) Western blots for decameric, dimeric and monomeric forms of PRDX-2 in lymphocyte cell lysates under non-reducing conditons $(n=4$; all men finished the race). PRE, POST and DAY 7 time points were selected to examine the most pronounced changes in PRDX-2 level. Over-oxidation of PRDX-2 can be seen immediately post-race as shown by a $25 \mathrm{kDa}$ band that is prominent in subjects 2 and 9, which is absent seven days later. B) PRDX-2 level represented as a ratio of over-oxidized to reduced/mildly oxidized PRDX-2. C) Over-oxidized and reduced/mildly oxidized PRDX-2 expressed as a percentage of all PRDX-2 oligomers present under non-reducing conditions.

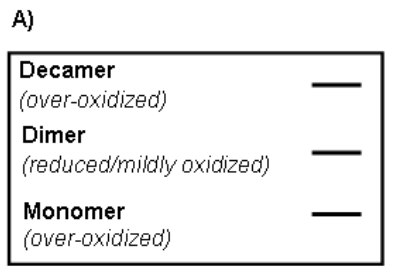

B)

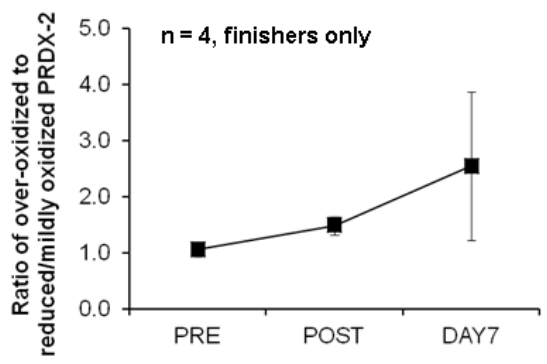

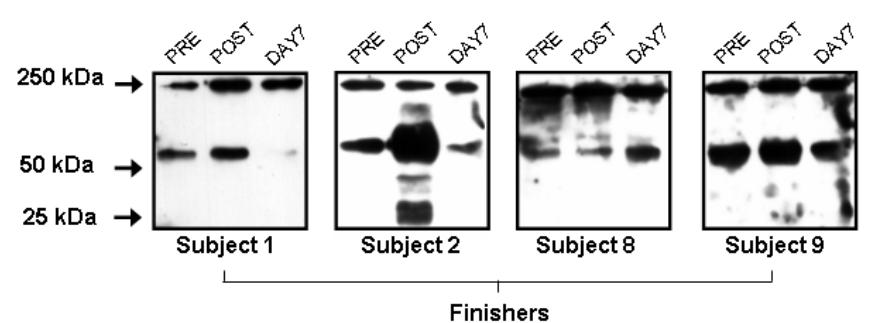

C)

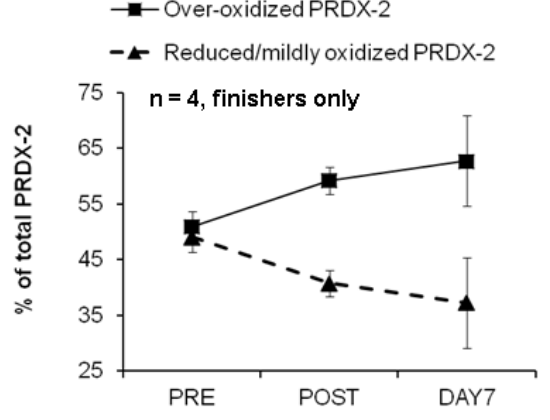

